\title{
Role of hepatobiliary scintigraphy with different semi-quantitative parameters along with histopathological scoring in differentiating biliary atresia from neonatal hepatitis
}

\author{
Nsreen R. A. Mohamadien', Rania Makboul ${ }^{2}$, Shereen M. Galal ${ }^{3}$ and Nadia M. Mostafa ${ }^{1 *}$
}

\begin{abstract}
Background: Biliary atresia (BA) and neonatal hepatitis $(\mathrm{NH})$ are the two major causes of neonatal cholestasis (NC). However, both conditions had entirely different therapeutic schedule and prognosis. Considering BA as a surgical emergency, it is pretty important to accurately differentiate the two entities. The aim of the study is to evaluate the diagnostic utility of hepatobiliary scintigraphy (HBS) using a semi-quantitative technique as well as 15-point histopathological scoring system in differentiating BA from $\mathrm{NH}$.

Results: The sensitivity, specificity, and overall accuracy of HBS in the diagnosis of BA was $90.5 \%, 80 \%$, and $83.6 \%$, respectively. The median values of kidney-liver ratio (KLR), intestinal-liver ratio (ILR), and background-liver ratio (BLR) were significantly higher in patients with BA, while that of the liver-kidney ratio (LKR) was significantly lower in cases with BA. Moreover, KLR had the largest area under curve (AUC); advocates it to be the best of the semiquantitative parameters that can predicts BA. Histopathological scoring using a cutoff point $\geq 7$ was helpful in discriminating BA from NH with $85.7 \%$ sensitivity, $95 \%$ specificity and $91.8 \%$ accuracy.

Conclusions: HBS is a non-invasive diagnostic tool frequently used in diagnosis of BA, yet it has a relatively low specificity. To overcome this challenge, we kindly recommend the use of semi-quantitative parameters that could possibly improve the accuracy of HBS for diagnosing BA. Additionally, the use of 15-point scoring for liver biopsy was useful.
\end{abstract}

Keywords: Biliary atresia, Radionuclide imaging, Cholestasis, Liver biopsy

\section{Background}

Neonatal cholestatic jaundice (conjugated hyperbilirubinemia) occurs when conjugated bilirubin is higher than $1 \mathrm{mg} / \mathrm{dl}$, if the total serum bilirubin is $\leq 5 \mathrm{mg} / \mathrm{dl}$, or $>$ $20 \%$ of total serum bilirubin when it is $>5 \mathrm{mg} / \mathrm{dl}$ [1]. Cholestasis is defined as reduced bile formation or flow through the intra- or extrahepatic biliary tree resulting

\footnotetext{
* Correspondence: nadia.khalifa@med.aun.edu.eg; drnadia1980@gmail.com ${ }^{1}$ Department of Clinical Oncology and Nuclear Medicine, Faculty of Medicine, Assiut University, Assiut, Egypt

Full list of author information is available at the end of the article
}

in accumulation of biliary substances within the liver. The incidence of neonatal cholestasis (NC) is approximately 1 in 2500 live births according to the recent guidelines for the evaluation of cholestatic jaundice in infants [2].

$\mathrm{NC}$ is always pathologic and signifies hepatobiliary disorder. Differential diagnoses of neonatal cholestatic jaundice include hepatocellular causes [neonatal hepatitis $(\mathrm{NH})$, metabolic diseases (galactosemia, tyrosinemia, alpha one antitrypsin deficiency, parenteral nutritionrelated cholestasis, and storage disorders)], ductal

\section{Springer Open}


paucity, and idiopathic and obstructive causes [biliary atresia (BA) and choledochal cyst] [3]. BA and $\mathrm{NH}$ are the two most common causes of neonatal cholestatic jaundice, yet they have similar clinical manifestations. The early correct discrimination of both conditions is mandatory for the outcome of BA and may avoid unnecessary surgery in cases with $\mathrm{NH}$ [4]. Hepatobiliary scintigraphy (HBS) using 99m TCiminodiacetic acid (IDA) derivatives is a widely used functional imaging modality for differentiating BA from $\mathrm{NH}$. The first and the frequently used radiopharmaceutical was $99 \mathrm{~m}$ TC-dimethyl IDA, which was referred to as hepatic IDA (HIDA). The radiotracer excreted by the liver in a similar manner to that of bilirubin into the bile ducts. So, HIDA scan relied on the principle that the presence of tracer in the bowel confirm patency of biliary channels and excludes BA [5-7].

A normal HBS shows rapid clearance of the radiotracer from the blood pool. Prompt and uniform hepatic uptake is reached within $5 \mathrm{~min}$, functional visualization of the gall bladder is seen as early as $10 \mathrm{~min}$ and bowel activity by $30-40 \mathrm{~min}[6]$.

The aim of the study was to assess the diagnostic accuracy of the semi-quantitative technique of HBS as a non-invasive diagnostic tool as well as the 15point histopathological scoring in differentiating BA from $\mathrm{NH}$ in correlation with histopathological diagnosis.

\section{Methods}

\section{Study population}

This retrospective study was approved by the Institutional Committee of Medical Ethics with a waiver of consent. Between January 2018 and December 2019, a total of 73 infants (mean age $79.62 \pm 32.8$ days, range 30-180 days) with cholestatic jaundice were referred to nuclear medicine unit in our institution for HIDA scintigraphy. Patients did not have liver biopsy or lost their histopathological data, as well as those with final diagnosis related to causes other than $\mathrm{NH}$ and $\mathrm{BA}$ were excluded leaving a valid cohort of sixty-one infants (37 boys and 24 girls) who were enrolled in this study. Cholestasis is considered if the serum direct bilirubin level was more than $1.0 \mathrm{mg} / \mathrm{dL}$ or $>17 \mathrm{mmol} / \mathrm{L}$ [2].

Detailed clinical history was obtained from mothers of all participants. All patients were subjected to complete physical examination and laboratory investigations including serum level of bilirubin (total and fractionated), complete blood count (CBC), prothrombin (PT) time, partial thromboplastin time (PTT), international randomization ratio (INR), and urine analysis. Percutaneous liver biopsy was performed for histopathological diagnosis after assurance of normal coagulation profile in addition to HIDA scan for all patients.

\section{Patient preparation and HBS protocol}

Pretreatment with phenobarbital (dose: $5 \mathrm{mg} / \mathrm{kg} /$ day for 5 days) to activate hepatic enzymes and increase bile flow in an attempt to reduce the risk of a false-positive results and maximize the specificity of the test in detection of BA. Patients were kept in fasting state for $2-4 \mathrm{~h}$ before the study (allowing only clear fluids if clinically indicated). After intravenous administration of ${ }^{99 \mathrm{~m}} \mathrm{Tc}-$ HIDA (200 uci $/ \mathrm{kg})$ into a peripheral vein, dynamic images were acquired in a rate of one frame every second for a total 60 frames, then one frame every minute for a total of 15 frames. Serial static images were acquired at $30 \mathrm{~min}, 1,2,4,6$, and $24 \mathrm{~h}$ after radiotracer injection, using a dual head SPECT/CT gamma camera (Symbia T, Siemens Healthcare, Erlangen, Germany) fitted with a low energy all-purpose parallel hole (LEAP) collimators, using $15 \%$ energy window set at $140 \mathrm{KeV}$ and matrix size of $128 \times 128$. The infants were imaged in a supine position, arms to the side and the imaging field included the heart, liver, intestine, and urinary bladder. All precautions were taken to avoid patient movement. The images were blindly reviewed by two experienced (15 and 11 years of experience) nuclear medicine physicians (the first reader conducted the visual analysis while the second reader performed the semiquantitative analysis).

\section{Qualitative analysis}

Patients without functional visualization of the gall bladder and/or bowel up $24 \mathrm{~h}$ were recorded as positive for BA. In contrast, those with functionally visualized gallbladder and/ or bowel (regardless of the timing or degree of excretion) $[5,6,8]$ were recorded as negative for BA. In addition, the time of blood pool clearance was estimated for all cases.

\section{Quantitative analysis}

Quantitative data readings were done by drawing a region of interest (ROI) on 30-min image frame over the liver and a mirror image ROI over the left kidney, paraumbilical region (intestinal uptake), and right thigh (background). The mean count in each ROI was obtained for estimation of liver-kidney ratio (LKR), kidneyliver ratio (KLR), intestinal-liver ratio (ILR), and background-liver ratio (BLR).

\section{Reference standard}

We relayed on the results of the histopathological examination as the reference standard as all of the included patients underwent liver biopsy. 


\section{Histopathological examination}

The paraffin-embedded block was sectioned and stained with Hematoxylin and Eosin stain. The biopsy materials were screened for adequacy in size and the number of portal tracts. Bile ductular proliferation, bile plugs in bile ductules, and porto-portal bridging are the standard histological features indicative of BA. The histological features indicative of $\mathrm{NH}$ includes lymphocytic and neutrophilic infiltration, lobular and portal inflammation and necrosis (lobular disarray), hepatocellular swelling, and giant cell transformation (Fig. 1).

\section{Histological scoring system}

The 15-point scoring system based on histology of liver biopsy, consisting of seven histological criteria to differentiate BA from $\mathrm{NH}$ was used. Each histological feature was scored and the sum of the scores was calculated. The score ranges from 0 to 15 points. Features against BA were negatively scored. A higher score was indicative of BA, while a lower score was less favorable for BA. A cut-off value score of $\geq 7$ had the best diagnostic utility to differentiate BA from other causes of intrahepatic cholestasis [9].

Bile ductular proliferation was considered to be present if the average number of ductules was more than five in the portal tract. The degree of bile ductular proliferation was scored as no proliferation-average number of bile ductules per portal tract $<5$; mild-average number of bile ductules per portal tract between 5 and 9; moderate- average number of bile ductules per portal tract $\geq 10$; and marked proliferation-attenuated, elongated, or angulated bile ductules in addition to proliferation (average number of bile ductules per portal tract $\geq 10$ ).

\section{Blinded histological interpretation}

The interpretation of the histology slides was done blindly without knowledge of the clinical data or the final clinical diagnosis. A blinded histological diagnosis was made for each histological specimen and was labeled as $\mathrm{BA}$ or $\mathrm{NH}$.

\section{Statistical analysis}

Data analysis was performed using SPSS version 21.0 (IBM Corp, Armonk, NY, USA). Based on histopathological examination, sensitivity, specificity, positive and negative predictive values, and diagnostic accuracy of ${ }^{99 \mathrm{~m}}$ Tc-HIDA scan were computed for BA versus $\mathrm{NH}$ and were compared with those of other imaging and laboratory investigations. Descriptive statistics of quantitative variables as mean $\pm \mathrm{SD}$, median (IQR) and range, as well as description of qualitative variables as number and percentage were used. The non-parametric MannWhitney test was used to compare medians between the two groups while independent sample $T$ test was used to compare mean between groups in normally distributed data. Chi-square test was used to compare qualitative variables. Area under the curve from ROC curve analysis

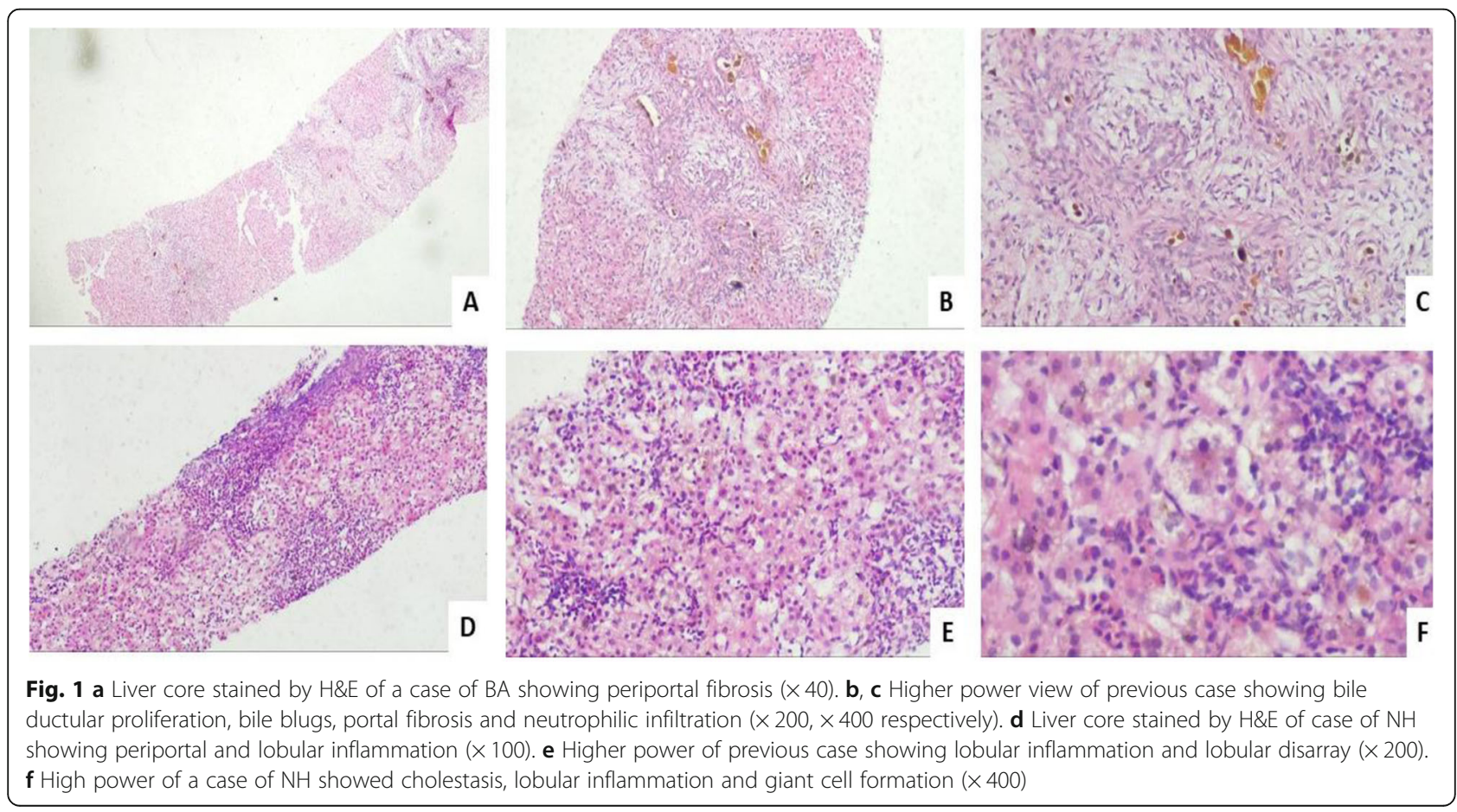


was used to evaluate the different ratios. Significant relationship was considered when $P$ value was $<0.05$

\section{Results}

Sixty-one infants with cholestatic jaundice were enrolled in this retrospective study in the period between January 2018 and December 2019, including 37 boys and 24 girls. The mean age at presentation was 79.62 days \pm 32.8 SD (range 30-180 days). The main clinical presentation in all infants was jaundice, other associated symptoms were acholic stool, dark urine, and fever in 18, 18, and 6 cases respectively. Mean serum direct bilirubin was $9.62(\mathrm{SD} \pm 23.71)$. Serological screening for TORCH syndrome was negative in 51 cases and positive in 10 cases. According to histopathological examination of liver biopsies, the final diagnosis of BA was established in $21(34.4 \%)$ infants while the other $40(65.6 \%)$ cases where diagnosed as $\mathrm{NH}$. The clinical and laboratory characteristics of the cases are illustrated in Table 1.

The mean total scoring was significantly higher for BA compared to NH group (9.1 vs. 3.8, $P<0.01)$. The histopathological scoring of both conditions was summarized in Table 2. Where portal duct proliferation, bile plug in portal tract, and porto-portal bridging were significantly more frequent in cases with BA $(P<0.001)$. Lymphocytic infiltrate in portal region, multinucleated hepatocyte, neutrophils in the infiltrate, and hepatocellular swelling were more frequent in cases with $\mathrm{NH}(P=$ $0.048,0.167,0.297$, and 0.176 respectively). Regarding the pathological diagnosis using 15-point scoring system, 41 cases were diagnosed as $\mathrm{NH}$ and 20 as BA. True positive and true negative findings were identified in 18/20 and $38 / 41$ cases respectively, giving $85.7 \%$ sensitivity, 95\% specificity, 92.7\% NPV, 90\% PPV, and 91.8\% accuracy (Chi-square 70.7, $P<0.01$ ).

Receiver operating characteristic [ROC] curve analyses showed a significant validity of histopathological scoring for diagnosis of BA (AUC: 0.949; SE: 0.028; 95\% CI: 0.893-1.000, $P<0.001$ ) (Fig. 2).

The final results of HIDA scintigraphy revealed BA in 27 infants and $\mathrm{NH}$ in the remaining 34 infants. Relying on histopathological examination, 19/27 and 32/34 cases were proved to be true positive (Figs. 3 and 4 ) and true negative (Fig. 5) respectively, giving 90.5\% sensitivity, $80 \%$ specificity, $70.4 \% \mathrm{PPV}, 94.1 \% \mathrm{NPV}$, and $83.6 \%$ accuracy (Chi-square 27.7, $P<0.001$ ). The time of blood pool clearance was significantly higher in cases with BA $(P=0.034)$. Using the semi-quantitative analysis of the HIDA scintigraphy, the median values of KLR, ILR, and BLR were significantly higher in cases with BA ( $P$ value $=0.008,0.011,0.016$ respectively) while that of the LKR was significantly lower in cases with BA $(P$ $=0.006$ ) (Table 3). Parameters which showed significant association with the diagnosis of BA were subjected to further analysis with ROC to determine which of them is the best to predict the diagnosis of BA. We found that KLR, ILR, BLR, and time of blood pool clearance were fairly predictive of BA with KLR had the largest AUC of 0.707 (95\% CI: 0.573-0.840, $P=0.008$ ), indicating that KLR was the best of the semi quantitative parameters in predicting BA (Fig. 6) (Table 4).

Table 1 Clinical and laboratory characteristics

\begin{tabular}{|c|c|c|c|}
\hline \multirow[t]{2}{*}{ Parameter } & \multicolumn{2}{|c|}{ Pathologic diagnosis } & \multirow{2}{*}{$\begin{array}{l}P \\
\text { value }\end{array}$} \\
\hline & $\begin{array}{l}\text { BA (21) } \\
\text { Number and \% }\end{array}$ & $\begin{array}{l}\mathrm{NH}(40) \\
\text { Number and \% }\end{array}$ & \\
\hline Age: & & & $0.152^{* *}$ \\
\hline • Mean (SD) & $70 \pm 30.5$ & $84.7 \pm 33.3$ & \\
\hline - Median(IQR) & $60(45)$ & $90(52.5)$ & \\
\hline Total bilirubin & & & $0.802^{* *}$ \\
\hline - Mean (SD) & $13 \pm 14.9$ & $10 \pm 6.5$ & \\
\hline - Median (IQR) & $8.05(7)$ & $8.65(11)$ & \\
\hline Direct bilirubin & & & $0.641^{* *}$ \\
\hline - Mean (SD) & $15 \pm 38.5$ & $6 \pm 4.2$ & \\
\hline • Median (IQR) & $6.5(5)$ & $5(7)$ & \\
\hline Sex & & & $0.684^{*}$ \\
\hline - Male & 12(57.1\%) & $25(62.5 \%)$ & \\
\hline • Female & $9(42.9 \%)$ & 15 (37.5\%) & \\
\hline Stool color & & & $0.635^{*}$ \\
\hline - Pigmented & $14(66.7 \%)$ & $29(72.5 \%)$ & \\
\hline - Acholic & 7 (33.3\%) & $11(27.5 \%)$ & \\
\hline Dark urine & & & $0.907^{*}$ \\
\hline - Present & $6(28.6 \%)$ & $12(30 \%)$ & \\
\hline - Absent & 15 (71.4\%) & $28(70 \%)$ & \\
\hline Liver size & & & $0.545^{*}$ \\
\hline - Normal & $11(52.4 \%)$ & $20(50 \%)$ & \\
\hline - Absent & $8(38.1 \%)$ & $12(30 \%)$ & \\
\hline - Not assessed & $2(9.5 \%)$ & $8(20 \%)$ & \\
\hline ICU admission: & & & $0.662^{*}$ \\
\hline - Yes & $8(38.1 \%)$ & $13(32.5 \%)$ & \\
\hline - No & $13(61.9 \%)$ & $27(67.5 \%)$ & \\
\hline Blood transfusion & & & $0.576^{*}$ \\
\hline - Yes & $3(15 \%)$ & $8(21 \%)$ & \\
\hline - No & $17(85 \%)$ & $30(79 \%)$ & \\
\hline Fever & & & $0.335^{*}$ \\
\hline - Present & $1(4.8 \%)$ & $5(12.5 \%)$ & \\
\hline - Absent & 20 (95.2\%) & 35 (87.5\%) & \\
\hline TORCH & & & $0.455^{*}$ \\
\hline - Positive & $5(23.8 \%)$ & $5(12.5 \%)$ & \\
\hline - Negative & $16(76.2 \%)$ & 35 (87.5\%) & \\
\hline
\end{tabular}

Statistical relationships were evaluated by Chi-square test*, Mann-Whitney test**. SD standard deviation 


\section{Discussion}

Through the many causes of persistent cholestatic jaundice in infancy, BA and $\mathrm{NH}$ account for $70-80 \%$ of cases [9]. Yet, both conditions had completely different therapeutic schedule and prognosis [10]. BA is a surgical emergency, Kasai portoenterostomy surgical treatment ideally performed in the first 60 days of life. If the surgery is not performed within 90 days, it will progress into liver cirrhosis and hepatic failure which is difficult to recover a normal condition [11]. On the other hand, conservative treatment is very effective in the management of almost all cases of $\mathrm{NH}$ [12]. So, it is pretty important to accurately differentiate BA from medical causes, mainly $\mathrm{NH}$.
However, no preoperative diagnostic algorithm has proven $100 \%$ diagnostic accuracy for BA [3].

Consistent with many previous studies [9, 11-15], the current study demonstrated that $\mathrm{NC}$ is more frequent in male patients (37 males vs. 24 females). Based on histopathological examination of liver biopsy, 21 cases (12 males and 9 females, 34.4\%) were diagnosed as BA while the remaining 40 cases ( 25 males and15 females, $65.6 \%$ ) were diagnosed as $\mathrm{NH}$. In keeping with other studies $[12,16,17]$, we found that BA tend to present at a relatively younger age compared to $\mathrm{NH}$ (mean age at the time of HBS was $70 \pm 30.5$ days for BA vs. $84.7 \pm 33.3$ days for $\mathrm{NH}$ ). On the other contrary, Zhou et al. found

Table 2 Histopathological scoring

\begin{tabular}{|c|c|c|c|}
\hline \multirow[t]{2}{*}{ Scoring parameter } & \multicolumn{2}{|c|}{ Pathologic diagnosis } & \multirow[t]{2}{*}{$P$ value } \\
\hline & $\begin{array}{l}\text { BA (21) } \\
\text { Number and \% }\end{array}$ & $\begin{array}{l}\mathrm{NH}(40) \\
\text { Number and \% }\end{array}$ & \\
\hline Portal duct proliferation: & & & $<0.001^{*}$ \\
\hline - None (0) & $1(4.8 \%)$ & $33(82.5 \%)$ & \\
\hline - Mild (1) & $2(9.5 \%)$ & $4(10 \%)$ & \\
\hline - Moderate (2) & $9(42.9 \%)$ & $3(7.3 \%)$ & \\
\hline - Marked (3) & $9(42.9 \%)$ & $0(0.0 \%)$ & \\
\hline Bile plug in portal ductules & & & $<0.001^{*}$ \\
\hline • Absent (0) & $5(23.8 \%)$ & $34(85 \%)$ & \\
\hline - Present (2) & $16(76.2 \%)$ & $6(15 \%)$ & \\
\hline Porto-portal bridging: & & & $<0.001^{*}$ \\
\hline • None $(0)$ & $3(14.3 \%)$ & $34(85 \%)$ & \\
\hline$\cdot<50 \%$ of portal tracts $(1)$ & $9(42.9 \%)$ & $5(12.5 \%)$ & \\
\hline$\cdot>50 \%$ of portal tracts (2) & $9(42.9 \%)$ & $1(2.5 \%)$ & \\
\hline Lymphocytic infiltrate in portal region & & & $0.048^{*}$ \\
\hline • None (2) & $0(0 \%)$ & $0(0 \%)$ & \\
\hline - Mild (1) & $14(66.7 \%)$ & $16(40 \%)$ & \\
\hline • Moderate/Sever (0) & 7 (33.3\%) & $24(60 \%)$ & \\
\hline Multinucleated hepatocyte & & & $0.167^{*}$ \\
\hline • None (2) & $9(42.9 \%)$ & $8(20 \%)$ & \\
\hline - Only around central vein (1) & $8(38.1 \%)$ & $21(52.5 \%)$ & \\
\hline • Diffuse (0) & $4(19 \%)$ & $11(27.5 \%)$ & \\
\hline Neutrophils in the infiltrate: & & & $0.297^{*}$ \\
\hline - Absent or mild (1) & $21(100 \%)$ & $38(95 \%)$ & \\
\hline - Moderate or marked (0) & $0(0.0 \%)$ & $2(5 \%)$ & \\
\hline Hepatocellular swelling & & & $0.176^{*}$ \\
\hline • None (2) & $6(28.6 \%)$ & $4(10 \%)$ & \\
\hline - Mild/focal (1) & $11(52.4 \%)$ & $26(65 \%)$ & \\
\hline - Periportal / diffuse (0) & $4(19 \%)$ & $10(25 \%)$ & \\
\hline Total score & & & $<0.01^{* *}$ \\
\hline Mean \pm SD & $9.1 \pm 2.5$ & $3.8 \pm 1.6$ & \\
\hline
\end{tabular}

Statistical relationships were evaluated by Chi-square test* and independent sample t-test** 
that both $\mathrm{BA}$ and $\mathrm{NH}$ groups had approximately the same age at the time of HBS [11]. We did not find a significant relationship between BA and acholic stool (reported in only $33.3 \%$ of BA cases, $P=0.635$ ). This comes in contrast to Abou-Taleb et al. and Dehghani et al. who observed a significant correlation (acholic stool was found in 100\% $(P=0.0001)$ and $95 \%(P=$ $0.003)$ of BA cases), respectively [12, 17]. However, similar to both of them, our study did not reveal a significant association between BA and the level of serum direct bilirubin. In agreement with relevant reports, we found that HBS had an overall accuracy of $83.6 \%$ in diagnosing BA $[12,18]$. Several studies reported variable degrees of sensitivity (84.6-100\%) and specificity (61.1-88.6\%) for the HBS in diagnosing BA [19-23]. In a large metaanalysis discussing the accuracy of $99 \mathrm{mTc}$-IDA HBS in differentiating BA from NH, Kianifar et al. have reported a pooled sensitivity of $98.7 \%$ and a pooled specificity of $70.4 \%$; the reported high sensitivity was due to the extremely rare false negative results recorded [24]. In the current study, HBS had a sensitivity of $90.5 \%$ and a specificity of $80 \%$ in diagnosing BA. We observed bowel activity in two cases ultimately diagnosed as BA (false negative for BA), at 30-min image in one case and 2-h image in the other (Fig. 7). Given the progressive obliterative nature of $\mathrm{BA}$, it is not surprising for HBS to show tracer excretion owing to the scan timing in respect to the degree of occlusion of extra-hepatic bile ducts. Muthukanagarajan et al. recorded that only $20 \%$ of BA showed complete fibroinflammatory obliteration [25].
This is matched with a recent case series study that reported biliary excretion in 6 infants finally diagnosed as BA. Despite the presence of tracer excretion, yet it was abnormal in all of the included infants (either delayed (6- and 24-h image) or subtle detected activity). Thus, they hypothesized that excluding BA relying on detection of any radioactivity in the intestine without consideration of the degree or timing of excretion is an erroneous conclusion that could result in delayed diagnosis and treatment of BA with subsequent inevitable negative impact on the outcome [26]. Kim et al., in a case report, found bowel activity at 4-h image during HBS for an infant with $\mathrm{NC}$, finally diagnosed to have BA. It was attributed to the presence of a remnant slit-like patent lumen in the pathological specimen that allowed bile/ tracer transit into the duodenum in this patient [27].

On the other hand, we encountered 8 cases with false positive results for BA in our study (did not demonstrate tracer excretion up to 24-h imaging despite final diagnosis of NH, Fig. 8). In part, this could be attributed to severe $\mathrm{NH}$ which was evident in 6 cases resulting in poor hepatocellular extraction, no tracer excretion, and consequently non-visualization of bowel. Low birth weight, prematurity, and total parenteral nutrition also decrease the capacity to excrete the radiotracer into the intestine, any of them could be another contributing factor [15]. However, due to the retrospective nature of our study, these clinical data were not amenable for us.

In the other part, co-existing mechanical obstruction of non-BA origin might be suggested in the remaining

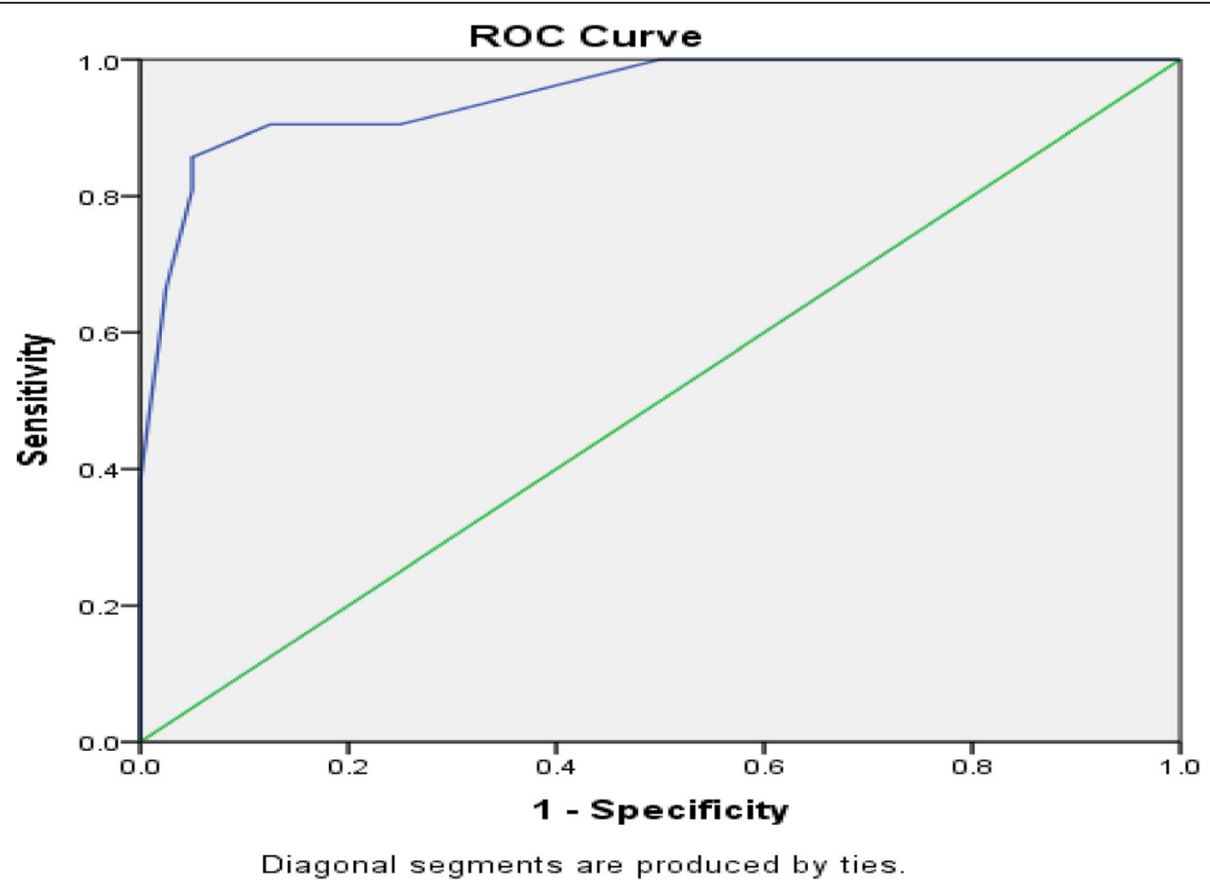

Fig. 2 Association between histopathological scoring and diagnosis of BA 


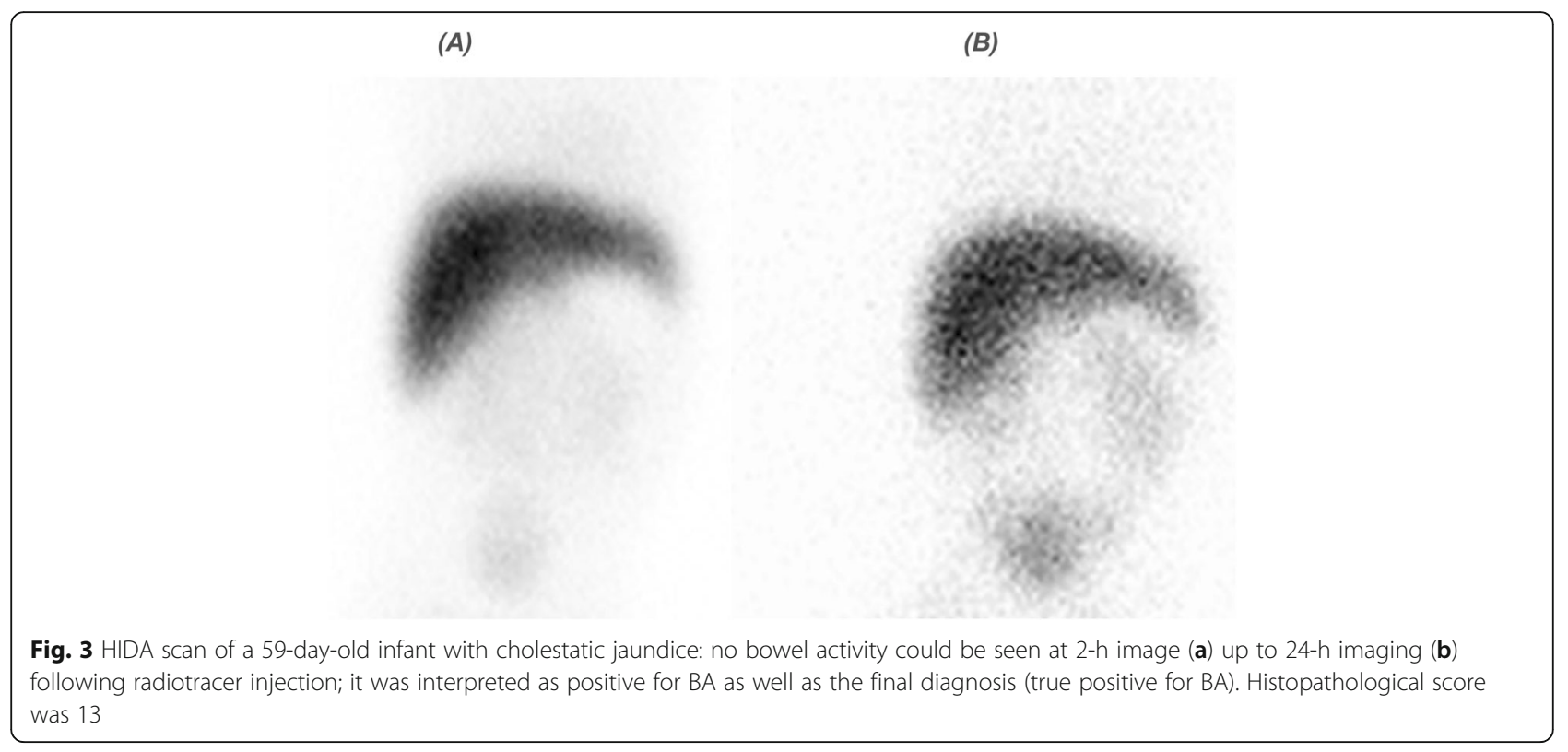

(A)

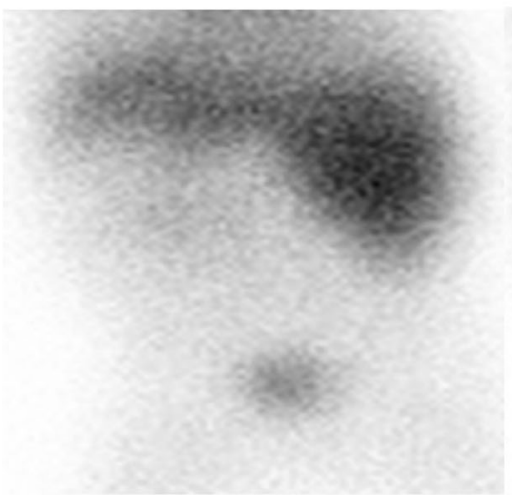

(C)

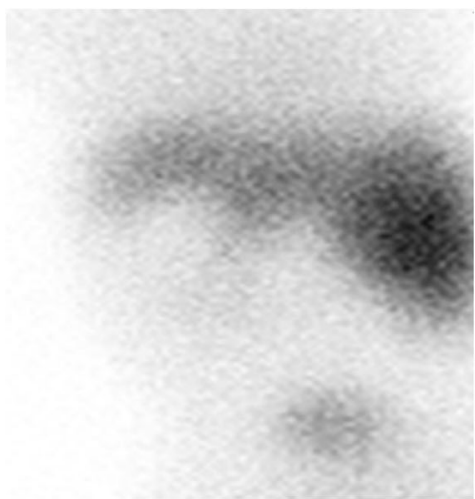

(B)

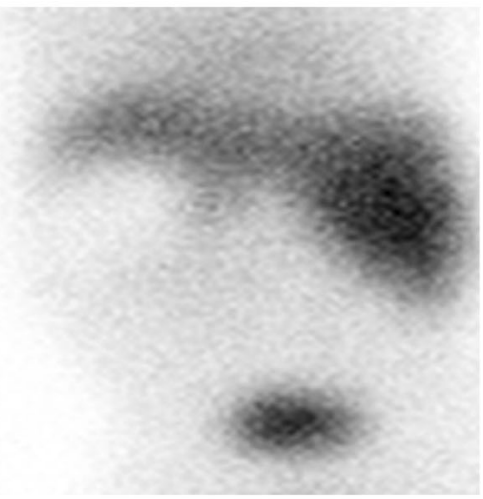

(D)

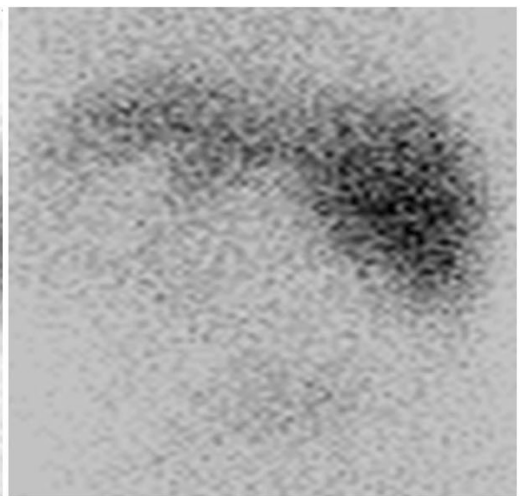

Fig. 4 HIDA scan of a 55-day-old infant with cholestatic jaundice: interestingly, this case incidentally discovered to have situs inversus during the examination. No bowel activity could be seen at 15-min (a), 1-h (b), 6-h (c) , and 24-h imaging (d) following radiotracer injection with evident renal clearance into urinary bladder, it was interpreted positive for BA as well as the final diagnosis (true positive for BA). Histopathological score was 10 
(A)

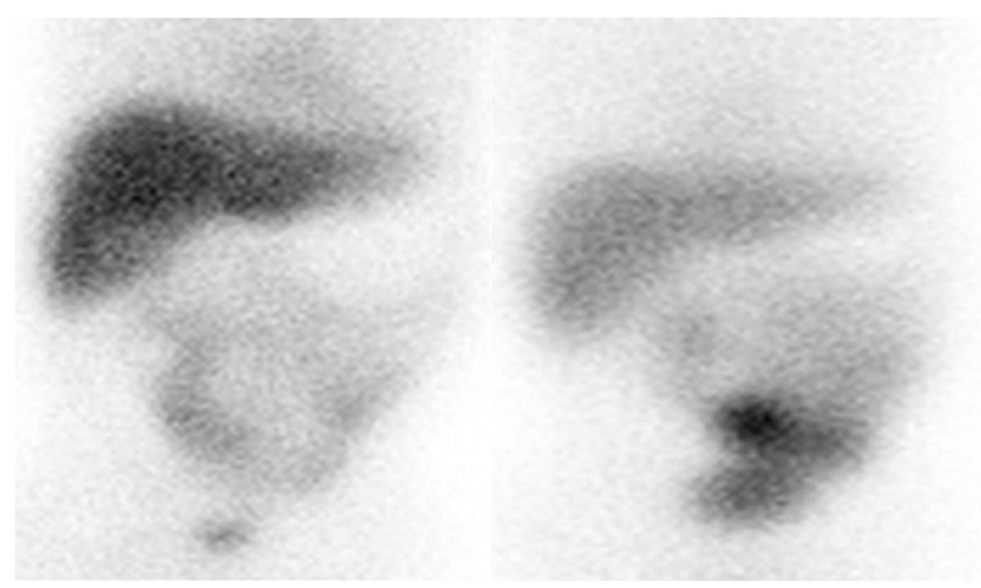

Fig. 5 HIDA scan of an 85-day-old infant with cholestatic jaundice: bowel activity was evident at 1-h image (a) which increased over time up to 4-h imaging (b) following radiotracer injection; it was interpreted negative for BA and finally diagnosed as NH (true negative for BA).

Histopathological score was 4 two cases, as both of them showed reasonable hepatic extraction with persistent hepatogram up to 24-h image. However, one infant had lost to follow up while the other infant died before further diagnostic interventional procedures were conducted. Brittain et al. falsely interpreted 4 cases as BA, finally proved to have mechanical obstruction of non-BA etiology on laparotomy [22].

Adding single-photon emission computed tomography/computed tomography (SPECT/CT) in cases of no or atypical drainage could be helpful for evaluating the non-BA origin. A previous study documented that

Table 3 Semiquantitative analysis of HIDA scan

\begin{tabular}{|c|c|c|c|}
\hline \multirow[t]{2}{*}{ Parameter } & \multicolumn{2}{|c|}{ Pathologic diagnosis } & \multirow{2}{*}{$\begin{array}{l}P \text { - } \\
\text { value }\end{array}$} \\
\hline & BA (21) & $\mathrm{NH}(40)$ & \\
\hline \multicolumn{4}{|c|}{ Blood pool clearance in min. } \\
\hline • Mean (SD) & $62.3 \pm 95.9$ & $31.8 \pm 66.8$ & $0.034^{* *}$ \\
\hline - Median (IQR) & $15(46)$ & $15(28)$ & \\
\hline \multicolumn{4}{|l|}{ LKR: } \\
\hline • Mean (SD) & $2.13 \pm 2$ & $4.8 \pm 4.1$ & $0.006^{* *}$ \\
\hline - Median(IQR) & $1.6(2)$ & $3.2(5.5)$ & \\
\hline \multicolumn{4}{|l|}{ KLR: } \\
\hline • Mean (SD) & $0.8 \pm 0.6$ & $0.48 \pm 0.5$ & $0.008^{* *}$ \\
\hline - Median(IQR) & $0.6(0.9)$ & $0.3(0.4)$ & \\
\hline \multicolumn{4}{|l|}{ ILR: } \\
\hline • Mean (SD) & $0.4 \pm 0.25$ & $0.3 \pm 0.25$ & $0.011^{* *}$ \\
\hline - Median(IQR) & $0.4(0.3)$ & $0.2(0.2)$ & \\
\hline \multicolumn{4}{|l|}{ BLR: } \\
\hline • Mean (SD) & $0.22 \pm 0.15$ & $0.14 \pm 0.1$ & $0.016^{* *}$ \\
\hline - Median(IQR) & $0.2(0.1)$ & $0.1(0.1)$ & \\
\hline
\end{tabular}

Mann-Whitney test** combining HBS with SPECT/CT resulted in improved specificity and accuracy in diagnosing BA [27].

As shown by the present study, the false-positive results are the major constraint for HBS. Accordingly, we aimed to retrospectively evaluate the diagnostic efficiency of the semi-quantitative approach of HBS in an attempt to improve the specificity in addition to reducing the examination time required to reach a correct diagnosis. We utilized semiquantitative methods to calculate LKR, KLR, ILR, and BLR. To our knowledge, no previous study has evaluated the last 3 parameters (KLR, ILR, and BLR) as semi-quantitative parameters of HBS for suspected BA. We found that all of them demonstrated high specificity (97.5\%) and sensitivity (95.5$100 \%$ ) in diagnosing BA. We noticed that LKR was significantly lower for BA group than for $\mathrm{NH}$ group $(P=$ 0.006), which comes in contrast to Liu et al. who used the same index to differentiate BA from $\mathrm{NH}$ and demonstrated that LKR of BA group was slightly higher than for NH group $(P<0.05)$ [28]. In the current study, significantly higher time of blood pool clearance noted in cases of BA $(P=0.034)$ could explain this un-expected finding which denoting impaired hepatic extraction efficiency with consequently alternative renal excretion. The higher mean of age (79.62 \pm 32.8 days) of our study population compared to that of Liu et al.'s study (45.9 \pm 23.4 days) might be the main underlying etiology for this discrepancy. Furthermore, a relatively small sample size and different IDA agent used in our study could be other aiding factors for different results.

In contrast, KLR, ILR, and BLR were significantly higher in cases with BA $(P=0.008,0.011$, and 0.016 respectively). It is worth noting that KLR had a higher AUC (0.707) than ILR and BLR (0.698 for both) 


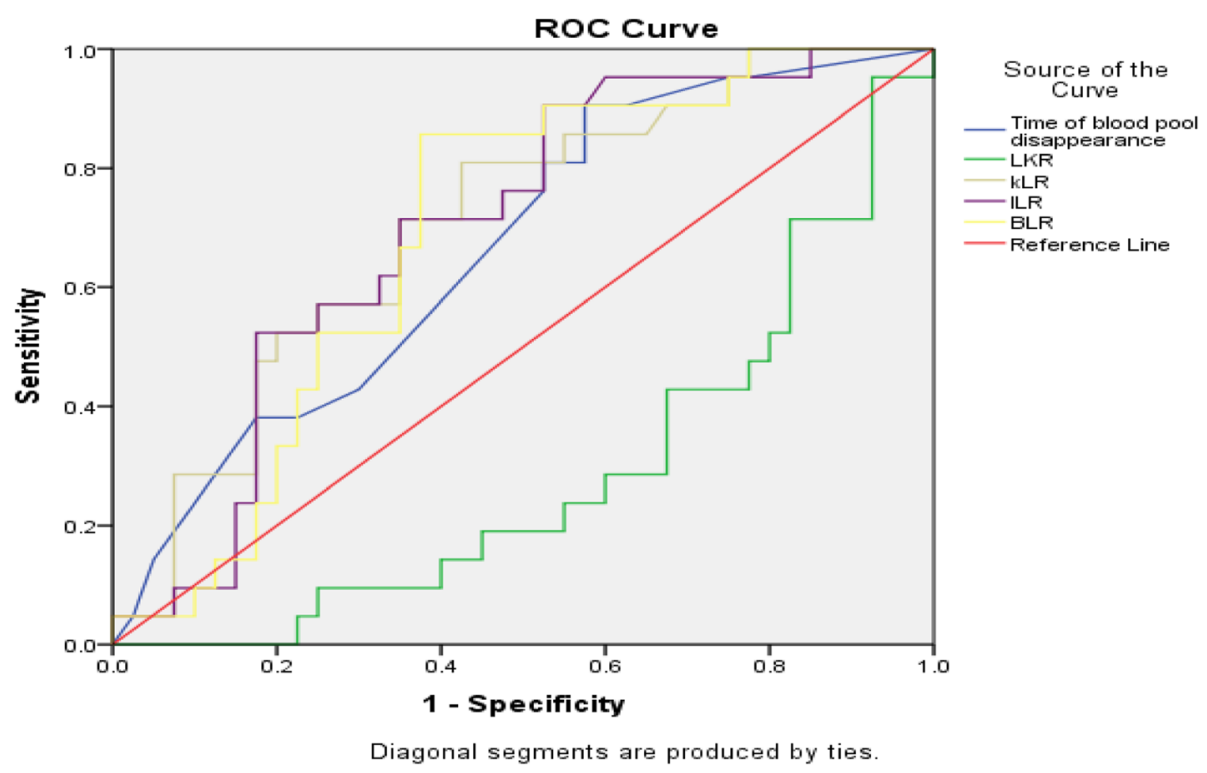

Fig. 6 Association between semiquantitative parameters and BA

indicating that KLR was the best semi-quantitative parameter to predict BA.

Regarding the 15-points histopathological scoring system, our results revealed that the median histopathological score to be 9 for $\mathrm{BA}$ and 3.5 for $\mathrm{NH}$, which is comparable to 10 for $\mathrm{BA}$ and 4 for $\mathrm{NH}$ in a recent similar study [15]. As mentioned above, we encountered a total of 10 false results for BA in our HBS evaluation. Two false negative results, both of them demonstrated high histopathological scoring $(\geq 7)$. On the other hand, 8 false positive results were reported, except for only one result had score $(\geq 7)$; the other 7 demonstrated low histopathological scoring $(<7)$. Comparable to the results of previous studies $[29,30]$, we found that histopathological scoring with a cutoff point $\geq 7$ had $85.7 \%$ sensitivity and $95 \%$ specificity for predicting BA. Also, Prasath Sathiah et al. reported a similar specificity (96\% vs. $95 \%$ ), yet a higher sensitivity (92\% vs. $85.7 \%$ ) for histopathological scoring. Different reported sensitivity may be attributable to different reference standard used for analysis; we relied on histopathological examination of liver biopsy as a reference standard while the gold standard in the other study was per-operative cholangiogram [15].

\section{Limitations}

Being a retrospective study, clinical data were not completely accessible. In addition, sonographic evaluation of the gall bladder as well as the serum level of gammaglutamyl transpeptidase which were previously reported as strong indicators for BA [12, 16, 31] were not available. On the other hand, the advantages of the current study included utilization of novel time saving semi-quantitative parameters in addition to histopathological scoring that was conducted by an experienced pathologist.

\section{Conclusion}

HBS is a non-invasive diagnostic tool, which is frequently used in diagnosis of BA. Despite its high sensitivity, the specificity of this imaging modality is relatively low. The time-saving semi-quantitative approach evaluated in our study had an incremental value in improving the diagnostic performance of $\mathrm{HBS}$ in diagnosing BA; we found that KLR was the best used novel parameter for this purpose. However, larger multicenter studies are needed before this approach can be applied. In addition, our findings confirmed the importance of a detailed histo-morphological evaluation of liver biopsy in all cases of NC.

Table 4 AUCs of the quantitative parameters

\begin{tabular}{|c|c|c|c|c|c|}
\hline Variable & AUC $(95 \% \mathrm{Cl})$ & Cut-off & Sensitivity & Specificity & $P$ value \\
\hline LKR & $0.286(0.154-0.417)$ & $\geq 0.49$ & 95.2 & 97.5 & 0.006 \\
\hline KLR & $0.707(0.573-0.840)$ & $\geq 0.063$ & 100 & 97.5 & 0.008 \\
\hline ILR & $0.698(0.565-0.831)$ & $\geq 0.048$ & 100 & 97.5 & 0.011 \\
\hline BLR & $0.698(0.556-0.823)$ & $\geq 0.027$ & 100 & 97.5 & 0.016 \\
\hline Time of blood pool clearance & $0.664(0.525-0.803)$ & $\geq 1.5 \mathrm{~min}$ & 95.2 & 77.5 & 0.036 \\
\hline
\end{tabular}


(A)

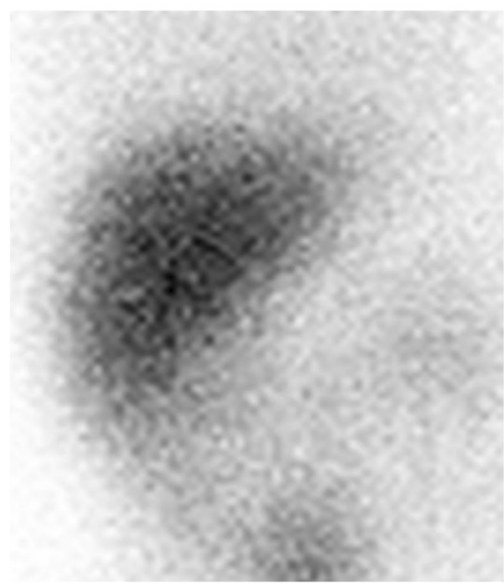

(B)

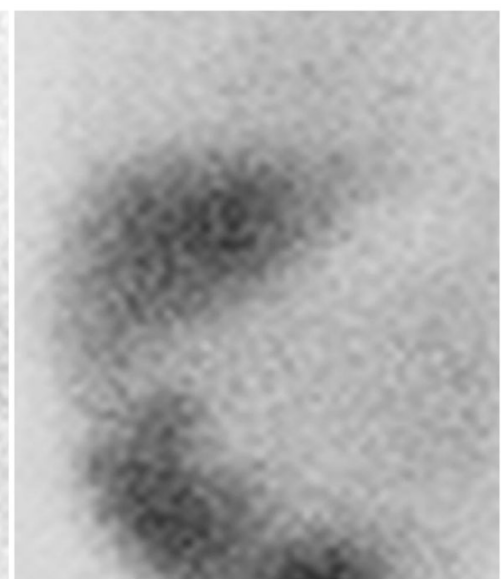

Fig. 7 HIDA scan of a 60-day-old infant with cholestatic jaundice: no bowel activity could be seen up to 1-h image (a), while evident tracer excretion into bowel was noted at 2-h imaging (b) following radiotracer injection. It was interpreted as negative for BA, yet finally diagnosed as BA (false negative for BA). Histopathological score was 10

(A)

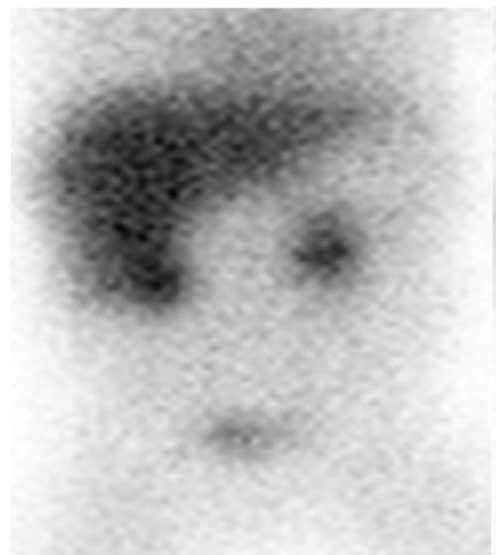

(C)

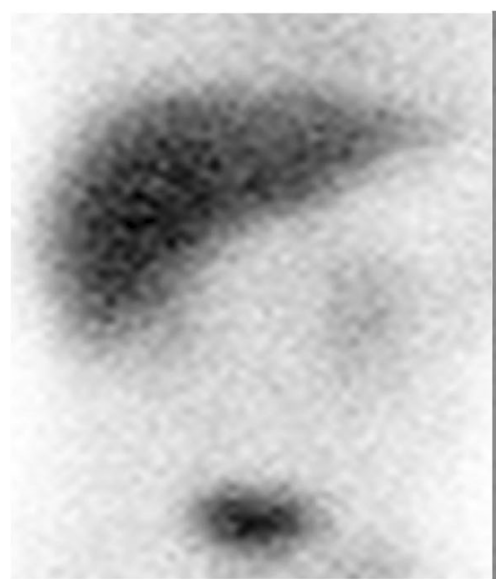

(B)

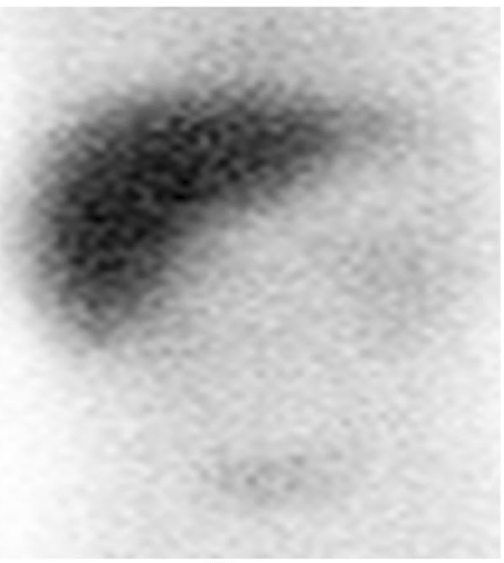

(D)

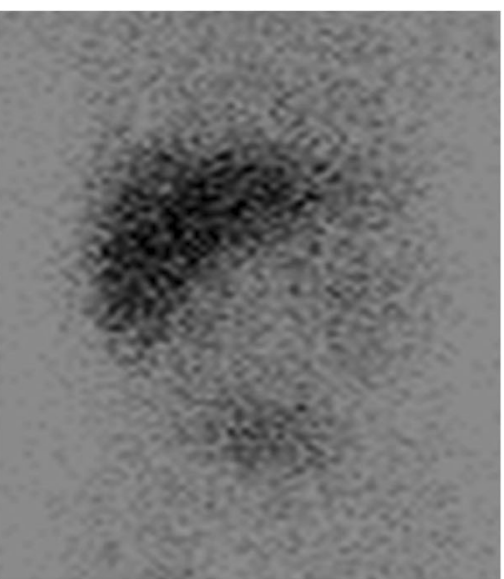

Fig. 8 HIDA scan of a 32-day-old infant with cholestatic jaundice: no bowel activity could be seen at 30-min (a), 4-h (b), 6-h (c), and 24-h imaging (d) following radiotracer injection. It was interpreted as BA, yet finally diagnosed as NH (false positive for BA). Histopathological score was 2 


\section{Abbreviations}

BA: Biliary atresia; $\mathrm{NH}$ : Neonatal hepatitis; NC: Neonatal cholestasis; HBS: Hepatobiliary scintigraphy; HIDA: Hepatobiliary iminodiacetic acid; LKR: Liver-kidney ratio; KLR: Kidney-liver ratio; ILR: Intestinal-liver ratio; BLR: Background-liver ratio; HIDA: Hepatic iminodiacetic acid

\section{Acknowledgements}

Not applicable.

\section{Authors' contributions}

Study concepts: NM, NR, RM, SM. Study design: NM, NR, RM. Data acquisition: NR, RM. Data analysis and interpretation: NM, NR, RM. Statistical analysis: NR. Manuscript preparation: NM, NR, RM. Manuscript editing: NM. Manuscript review: NM, NR, RM, SM. All authors read and approved the final manuscript.

\section{Funding}

No external funding was obtained for this study.

\section{Availability of data and materials}

The datasets used and analyzed during the current study are available from the corresponding author on reasonable request.

\section{Declarations}

\section{Ethics approval and consent to participate}

This study was performed in line with the principles of the Declaration of Helsinki. Approval was granted by the Ethics Committee of faculty of medicine, Assiut University, Egypt, with IRB number 17300342. Informed consent was waived due to the retrospective nature of the study.

\section{Consent for publication}

No separate individual consent was obtained from patients' parents for this study as it was retrospective in nature, and the examinations were carried out as part of routine clinical care. No patient-identifying information is included in the manuscript material.

\section{Competing interests}

The authors declare that they have no competing interest.

\section{Author details}

${ }^{1}$ Department of Clinical Oncology and Nuclear Medicine, Faculty of Medicine, Assiut University, Assiut, Egypt. ²Department of Pathology, Faculty of Medicine, Assiut University, Assiut, Egypt. ${ }^{3}$ Department of Pediatrics, Faculty of Medicine, Assiut University, Assiut, Egypt.

Received: 20 January 2021 Accepted: 30 March 2021

Published online: 16 April 2021

\section{References}

1. De Bruyne R, Van Biervliet S, Vande VS, Van Winckel M (2011) Clinical practice Eur J Pediatr 170(3):279-284. https://doi.org/10.1007/s00431-010-1363-8

2. Fawaz R, Baumann U, Ekong U, Fischler B, Hadzic N, Mack CL et al (2017) Guideline for the evaluation of cholestatic jaundice in infants: joint recommendations of the north American Society for Pediatric Gastroenterology, Hepatology, and nutrition and the European Society for Pediatric Gastroenterology, Hepatology, and nutrition. J Pediatr Gastroenterol Nutr 64(1):154-168

3. Mandelia A, Lal R, Mutt N (2017) Role of hepatobiliary scintigraphy and preoperative liver biopsy for exclusion of biliary atresia in neonatal cholestasis syndrome. Indian J Pediatr 84(9):685-690. https://doi.org/10.1 007/s12098-017-2408-Z

4. He J-P, Hao Y, Wang X-L, Yang X-J, Shao J-F, Feng J-X (2016) Comparison of different noninvasive diagnostic methods for biliary atresia: a meta-analysis. Springer

5. Cistaro A, Massollo M (2016) Nuclear medicine in pediatric gastrointestinal diseases. In: Clinical nuclear medicine in pediatrics. Springer, pp 149-171

6. Anand SS, Handa RK, Singh J, Sinha I (2006) Hepato-biliary scintigraphy in diagnosis of biliary atresia. Med J Armed Forces India 62(1):20-21. https:// doi.org/10.1016/S0377-1237(06)80146-2

7. Elgazzar AH, Sarikaya I (2018) Hepatobiliary system. In: Nuclear medicine companion. Springer, pp 203-217
8. Krebs S, Dunphy M (2017) Role of nuclear medicine in diagnosis and management of hepatopancreatobiliary disease. In: Blumgart's surgery of the liver, biliary tract and pancreas, 2-volume set. Elsevier, pp 285-315

9. Sultana N, Jabin Z, Bashir M, Parveen R, Begum SMF, Begum R et al (2015) Role of hepatobiliary scintigraphy and ultrasonography in the diagnosis of biliary atresia in infant with neonatal jaundice-experiences in NINMAS. Bangladesh J Nucl Med 18(1):51-53

10. Rebollar-Rangel JA, Escobedo-Torres P, Flores-Nava G (2017) Etiology of neonatal jaundice in children admitted for phototherapy. Rev Mex Pediatría 84(3):88-91

11. Zhou H, Li J, Wei H, Li A, Wang X, Zhang X, Lu Q, Chen S, Wei Z (2020) A novel timesaving and semiquantitative method for radionuclide hepatobiliary scintigraphy for suspected biliary atresia. Ann Palliat Med 9(1): 63-69. https://doi.org/10.21037/apm.2019.12.07

12. Abou-Taleb A, Abdelhameed W, Ahmed A, El-Hennawy A (2019) Noninvasive diagnostic methods for differentiation of biliary atresia from neonatal hepatitis in upper Egypt. Int J Pediatr 7(4):9265-9275

13. Sathiah P, Basu D, Kar R, Jagadisan B, Kumaravel S (2018) Evaluation of liver biopsies using histopathological scoring system in neonatal hepatitis and biliary atresia: correlation with clinico-radiological and biochemical parameters. J Clin Diagn Res 12(2):15-19

14. Yang J-G, Ma D-Q, Peng Y, Song L, Li C-L (2009) Comparison of different diagnostic methods for differentiating biliary atresia from idiopathic neonatal hepatitis. Clin Imaging 33(6):439-446. https://doi.org/10.1016/j.clinimag.2009.01.003

15. Dehghani SM, Haghighat M, Imanieh MH, Geramizadeh B (2006) Comparison of different diagnostic methods in infants with cholestasis. World J Gastroenterol: WJG 12(36):5893-5896. https://doi.org/10.3748/wjg.v12.i36.5893

16. Dong C, Zhu H, Chen Y, Luo X, Huang Z (2018) Clinical assessment of differential diagnostic methods in infants with cholestasis due to biliary atresia or non-biliary atresia. Curr Med Sci 38(1):137-143. https://doi.org/10.1 007/s11596-018-1857-6

17. Donia AES, Ibrahim SM, Kader M, Saleh AM, El-Hakim MS, El-Shorbagy MS et al (2010) Predictive value of assessment of different modalities in the diagnosis of infantile cholestasis. J Int Med Res 38(6):2100-2116. https://doi. org/10.1177/147323001003800626

18. Ghazy RM, Adawy NM, Khedr MA, Tahoun MM (2018) Biliary atresia recent insight Egypt Pediatr Assoc Gaz 66(1):1-8. https:/doi.org/10.1016/j.epag.2017.12.001

19. Kianifar HR, Tehranian S, Shojaei P, Adinehpoor Z, Sadeghi R, Kakhki VRD, Keshtgar AS (2013) Accuracy of hepatobiliary scintigraphy for differentiation of neonatal hepatitis from biliary atresia: systematic review and meta-analysis of the literature. Pediatr Radiol 43(8):905-919. https://doi.org/10.1007/s00247-013-2623-3

20. Muthukanagarajan SJ, Karnan I, Srinivasan P, Sadagopan P, Manickam S (2016) Diagnostic and prognostic significance of various histopathological features in extrahepatic biliary atresia. J Clin Diagn Res JCDR 10(6):EC23

21. Adeyemi A, States L, Wann L, Lin HC, Rand EB (2020) Biliary excretion noted on hepatobiliary iminodiacetic acid scan does not exclude diagnosis of biliary atresia. J Pediatr (220):245-248

22. Kim H, Park S, Ha S, Kim JS, Kim DY, Oh M (2019) False-negative hepatobiliary scintigraphy for biliary atresia. Nucl Med Mol Imaging 53(5): 356-360. https://doi.org/10.1007/s13139-019-00606-w

23. Brittain JM, Kvist N, Johansen LS, Borgwardt L (2016) Hepatobiliary scintigraphy for early diagnosis of biliary atresia. Dan Med J 63:A5253

24. Sevilla A, Howman-Giles R, Saleh H, Trpezanovski J, Concannon R, Williams K, Chung D, Uren R (2007) Hepatobiliary scintigraphy with SPECT in infancy. Clin Nucl Med 32(1):16-23. https://doi.org/10.1097/01.rlu.0000249860.41139.a6

25. Liu X, Qi-Lian R, Shi-Liang Z, Jun-Hong L, Zhi-Xiao W (2018) The value of 99mTc-EHIDA hepatobiliary scintigraphy with different liver/kidney ratio in the distinguish of infant persistent jaundice. J Med Diagn Meth 7(267):2

26. Krishna OHR, Sultana N, Malleboyina R, Kumar AN, Reddy KR, Rao BN (2014) Efficacy of the seven feature, fifteen point histological scoring system and CD56 in interpretation of liver biopsies in persistent neonatal cholestasis: a five-year study. Indian J Pathol Microbiol 57(2):196. https://doi.org/10.4103/0377-4929.134662

27. Lee WS, Looi LM (2009) Usefulness of a scoring system in the interpretation of histology in neonatal cholestasis. World J Gastroenterol: WJG 15(42): 5326-5333. https://doi.org/10.3748/wjg.15.5326

28. Chen X, Dong R, Shen Z, Yan W, Zheng S (2016) Value of gamma-glutamyl transpeptidase for diagnosis of biliary atresia by correlation with age. J Pediatr Gastroenterol Nutr 63(3):370-373. https://doi.org/10.1097/MPG.0000000000001168

\section{Publisher's Note}

Springer Nature remains neutral with regard to jurisdictional claims in published maps and institutional affiliations. 